\title{
OVERACTIVE BLADDER
}

\author{
Yordanov Delyan, \\ Dr., MD, Specialist Obstetrics and Gynecology, UMBAL "Deva Maria", Burgas \\ Yordanova Lilyna, \\ Dr., MD, Specialist Obstetrics and Gynecology, Bulgaria, Rousse, Kanev University Hospital
}

DOI: https://doi.org/10.31435/rsglobal_ws/30042020/7018

\section{ARTICLE INFO}

Received: 14 February 2020

Accepted: 10 April 2020

Published: 30 April 2020

\section{KEYWORDS}

overactive bladder syndrome, urinary incontinence, nocturia, detrusor overactivity, mirabegron.

\begin{abstract}
In recent years, there has been a steady increase in patients visiting a specialist for what is known as overactive bladder syndrome. Due to the extremely negative impact of the disease on patients' quality of life, timely diagnosis and adequate treatment choices are of the utmost importance. The guidelines presented include a description of the etiology and pathogenesis, basic principles of diagnosis, and current methods of treatment for this category of patients.
\end{abstract}

Citation: Yordanov Delyan, Yordanova Lilyna. (2020) Overactive Bladder. World Science. 4(56), Vol.1. doi: 10.31435/rsglobal_ws/30042020/7018

Copyright: (C) 2020 Yordanov Delyan, Yordanova Lilyna. This is an open-access article distributed under the terms of the Creative Commons Attribution License (CC BY). The use, distribution or reproduction in other forums is permitted, provided the original author(s) or licensor are credited and that the original publication in this journal is cited, in accordance with accepted academic practice. No use, distribution or reproduction is permitted which does not comply with these terms.

Introduction. Overactive bladder $(\mathrm{OAB})$ is a widespread syndrome. According to the International Continence Society (ICS), 17\% of Europe's adults have symptoms of OAB (Wein A.J., Rovner E.S., 2002). It should be noted that OAB without urinary incontinence (OAB-dry) is observed in $7.6 \%$ of women, and $\mathrm{OAB}$ in combination with urgency incontinence (OAB-wet) - in 9.3\%. There is currently a tendency to increase the incidence of $\mathrm{OAB}$ and urinary incontinence in women at age 65 and older. $\mathrm{OAB}$ is generally more frequent in women after 44 years (Stewart W.F., Van Rooyen, 2003). There is no clear data on the spread of OAB in Bulgaria, but it is generally accepted that it is similar to that in European countries.

Despite the fact that OAB is diagnosed more often in the elderly, its symptoms are also found in other age groups. Most patients are over 40 years of age. On the other hand, in men over 60 years there is a clear tendency to increase the incidence of the disease, and in women, on the contrary, decrease. Thus, OAB is a fairly common clinical syndrome that occurs in different age groups and leads to physical and social maladaptation.

Discussion. It is well established that neurogenic and non-neurogenic factors are the basis for the development of OAB. Neurogenic disorders occur at the level of the supra-spinal centers of the nervous system and spinal cord pathways, and non-neurogenic disorders occur due to age-related changes in detrusor, intravesical obstruction, and anatomical changes in urethral and bladder position. There are currently 3 main theories about $\mathrm{OAB}$.

○ Decreased central inhibitory effect on urination reflex. For example, as a result of a disorder of the blood supply to the brain (stroke);

○ Damage to axonal pathways and loss of peripheral inhibitory effects in spinal cord injury or multiple sclerosis;

- Identification of neurotransmission processes in reflex junctions leads to detrusive hyperactivity corresponding to sphincter insufficiency and insufficient suppression of urinary reflex due to a decrease in volitional control of the act of urination (non-inhibitory neurogenic bladder).

The spontaneous generation of action potential is quite limited in the bladder muscle, but with its partial denervation, the action potential can be transmitted from cell to cell, causing microcontraction in the smooth muscle cells of the detrusor, leading to increased bladder pressure and stimulation. of afferent 
detrusor receptors. The trigger can also be damage to the inhibitory pathways in the central nervous system, sensitization of peripheral afferent nerve endings and denervation due to age-related changes or peripheral neuropathy in diabetes mellitus, ischemia of bladder smooth muscle cells.

There are many risk factors for $\mathrm{OAB}$, the most common of which are age, smoking, obesity, consumption of carbonated and caffeinated beverages, reduced consumption of vegetables, fruits and bread, increased consumption of beef, sugar (Zhang W., 2006; Gormley E.A., Lightner D.J., 2015).

Urinary tract infection and overactive bladder

Urges and urgent urinary incontinence often occur in the presence of urinary infection. Speaking of $\mathrm{OAB}$, we should automatically exclude the presence of urinary tract infection. The urgent symptoms of cystitis cannot be regarded as $\mathrm{OAB}$, they are symptomatic urge incontinence or symptomatic urgency caused by inflammation of the bladder mucosa.

There is evidence that chronic inflammatory diseases of the bladder lie in the pathogenesis of $\mathrm{OAB}$. The first step may be the loss of mucosal glycosaminoglycans of the mucosal layer, with subsequent disruption of bladder function, manifested by increased contractility of the detrusor (Damiano R., Cicione A., 2011). In the presence of recurrent infectious and inflammatory processes, the biologically active substances involved in the inflammatory reaction act on certain receptors, thereby contributing to the development of pathological hypersensitivity of the bladder due to C-fiber irritation (Siracusano S., 2009, Cucchi, S., Cucchi, S., Cucchi, 2009).

History taking

A detailed medical history is needed: data for conservative, drug and / or surgical treatment to determine their efficacy and side effects.

Particular attention should be paid to the patient's age at the time of onset of symptoms, the presence of bowel disorders, sexual function and the nervous system.

It is necessary to clarify the family history, obstetric and gynecological history, as well as the presence of brain and spinal cord injuries, the presence of symptoms of neurological diseases (dizziness, tinnitus, impaired coordination), which may be accompanied by symptoms of OAB. It is important to understand what medicines the patient is taking for other ailments - whether these medicines contribute to urinary incontinence.

Laboratory diagnostics: Urinalysis

The diagnosis of $\mathrm{OAB}$ involves two stages. In the first stage, the exclusion of other diseases that occur with urgent urination is performed. In the second stage, the type of the OAB is determined. The initial examination of a patient with suspected $\mathrm{OAB}$ should include a urinalysis to exclude acute and chronic bladder and urethral disorders. Bladder hypersensitivity to inflammation of the lower urinary tract may be a cause of OAB-specific complaints.

Laboratory diagnostics: blood tests

The standard biochemical blood test for the determination of creatinine and urea levels has important diagnostic value for assessing the condition of the urinary system. Blood glucose is also important. Elevated glucose levels indicate the possible presence of diabetes mellitus, which is accompanied by polyuria, leading to frequent urination during the day and night.

Menopausal women often have a decrease in blood estrogen. Itching and burning in the genitals with hypoestrogenemia are not uncommonly accompanied by frequent urination, which may be mistaken for hyperactivity. However, sex hormone determination is not required in routine practice.

Gynecological examination

Gynecological examination of the vagina and cervix is required in women with suspected OAB. In this case, attention should be paid to the pelvic floor muscles, their tone, the anatomical position of the anterior and posterior walls of the vagina and uterus, and their ability to contract, which may also be the cause of OAB. A cough test is also performed to detect involuntary detrusor contractions caused by increased intra-abdominal pressure or accompanying urinary incontinence.

Genital prolapse is a common cause of urinary disorders. Prolapse of the anterior wall of the vagina or uterus can lead to intravesical obstruction and urge symptoms. Very often, the prolapse of the vaginal walls, especially the anterior wall, causes inflammation of the bladder, which contributes to the appearance of symptoms of overactivity. Pelvic disorders, including pelvic descent and prolapse, as well as urinary incontinence, are disabling conditions that lead to surgical treatment in 1 in 9 women (Hubchev, G., 2017)

Neurological examination

In the presence of a neurological disease that may be the cause of the symptoms of the lower urinary tract, neurogenic $\mathrm{OAB}$ should be discussed. 
Indications for Cystoscopy

Endoscopic examination of the lower urinary tract is recommended in the following cases:

- another pathology, such as haematuria, was detected during the initial examination;

- in the presence of pain or discomfort in the bladder - in patients with $\mathrm{OAB}$, endoscopy can reveal an intravesical formation (stones, tumors), which can cause dysuria;

- exclusion of extraurethral urinary incontinence or stress urinary incontinence, if necessary.

Urethrocystoscopy examines the urethra and bladder for organic diseases, lesions and concrements. The response of urethral sphincter mechanisms and detrusor in response to bladder filling is evaluated. In women with stress incontinence, the bladder neck dilates when the bladder is filled, during straining and coughing, while at the compression command, the reaction is slow or absent. (G. Hubchev, 2017)

\section{Ultrasound indications and its characteristics}

Visualization of the lower urinary tract and pelvic organs is recommended in cases where concomitant pathology in this area is suspected during the initial examination. The initial stage may be ultrasound or X-ray examination.

The determination of residual urine is recommended by ICS and is a routine screening test for all patients with urinary incontinence. The most commonly practiced is abdominal ultrasound.

Urodynamic studies allow assessment of the condition of the lower urinary tract and formulate an additional plan for examination and treatment of the patient.

Non-invasive tests include all urodynamic tests performed without the introduction of catheters or sensors (uroflowmetry, determination of residual urine volume).

Invasive urodynamic testing (cystometry) is the standard for diagnosing the detrusor hyperactivity. According to cystometry data, the filling account for: increase in the sensitivity of the bladder, decrease in its elasticity and values of the so-called compliance (the ratio of the change in bladder volume to detrusor pressure).

\section{Treatment}

Behavioral therapy in the treatment of $\mathrm{OAB}$ is aimed at forming a new model of urination or restoration of the previous one, in which this process becomes controlled by the patient again (Ahlberg J., 2002).

\section{- Correction of fluid intake regime}

- Bladder training, exercise and biofeedback therapy

\section{- Medication treatment}

Antimuscarinic preparations (m-anticholinergic drugs) are currently the basis of treatment for $\mathrm{OAB}$ and urgent urinary incontinence. (Hubchev G., 2020). They differ in their pharmacological characteristics, for example, affinity for muscarinic acetylcholine receptors and other forms of action, in their pharmacokinetic properties, for example, lipid solubility, half-life.

Oxybutynin; Tolterodine; Trospium chloride; Solifenacin; $\beta$-3-Adrenomimetics - Mirabegron; Vasopressin analogues - Desmopressin

\section{- Intravesical injections of botulinum toxin type A}

Intravesical administration of botulinum toxin type A at a dose of 100 units is a common method of treating $\mathrm{OAB}$ syndrome with persistent or refractory to $\mathrm{m}$-anticholinergic therapy in women with urinary incontinence. In clinical studies, a single intravesical injection of botulinum toxin type A was shown to be more effective than the placebo effect, reducing the severity of urge incontinence symptoms, improving the patient's quality of life for 12 months (Vij M., Drake MJ, 2015 ). However, treatment is repeated on every 4-8 months. There is no evidence of a decrease in the efficacy of type A botulinum toxin after repeated injection.

\section{- Neuromodulation}

The main difference between neuromodulation and other stimulation methods is the following: the effect manifests itself on nerve roots or peripheral nerves (in the case of tibial stimulation).

\section{- Urinary bladder augmentation and urine derivation}

Augmentation cystoplasty can only be offered to patients with refractory detrusor hyperactivity and urinary incontinence in case of unsuccessful conservative treatment, botulinum toxin injection and sacral electrical stimulation. The main indication for surgical treatment is a significant reduction in the anatomical capacity of the bladder. Patients who are offered cystoplasty augmentation or urinary derivation should be advised of the high risk of periodic bladder self-catheterization as well as of early and late complications and of a slightly increased risk of bowel cancer. 
Conclusions. Modern urogynecology is aimed at providing high quality of life for patients. In this regard, particular attention is paid to the treatment of those uro-gynecological conditions that directly impair women's quality of life. $\mathrm{OAB}$ is a syndrome consisting of many symptoms (urgency, urge incontinence, frequent urination and nocturia).

- Only urgency is a mandatory symptom of OAB. In order to be diagnosed, the specialist must rule out all other possible reasons for explaining the patient's complaints.

- Urodynamic testing should be performed in situations where behavioral therapy and first-line treatment do not help.

- Cystoscopy is indicated for women who complain of hematuria or painful urination.

- Limiting your coffee or tea intake can very often eliminate or reduce your symptoms of OAB.

- M-anticholinergics - first-line medicines for the treatment of OAB and urinary incontinence.

- If the prescribed m-anticholinergic drug does not have sufficient therapeutic effect, another $m$-anticholinergic antagonist can be used and the dose increased to its maximum.

- $\beta$-3-Adrenomimetic mirabegron may be prescribed to patients who have pronounced side effects from the use of $m$-anticholinergics, as well as to those patients who are not helped by $m$-anticholinergics.

- Dose duplication of $\mathrm{m}$-anticholinergics is acceptable in women with neurogenic urinary disorders.

- Botulinum toxin for intravesical administration should be used in patients refractory to oral medicines. A dose of 100 units does not lead to a significant risk of urinary retention, and bladder infection is the most common side effect.

- Augmentation of the bladder or the creation of a bowel reservoir is indicated in situations of dramatic reduction of the anatomical capacity of the bladder and total urinary incontinence.

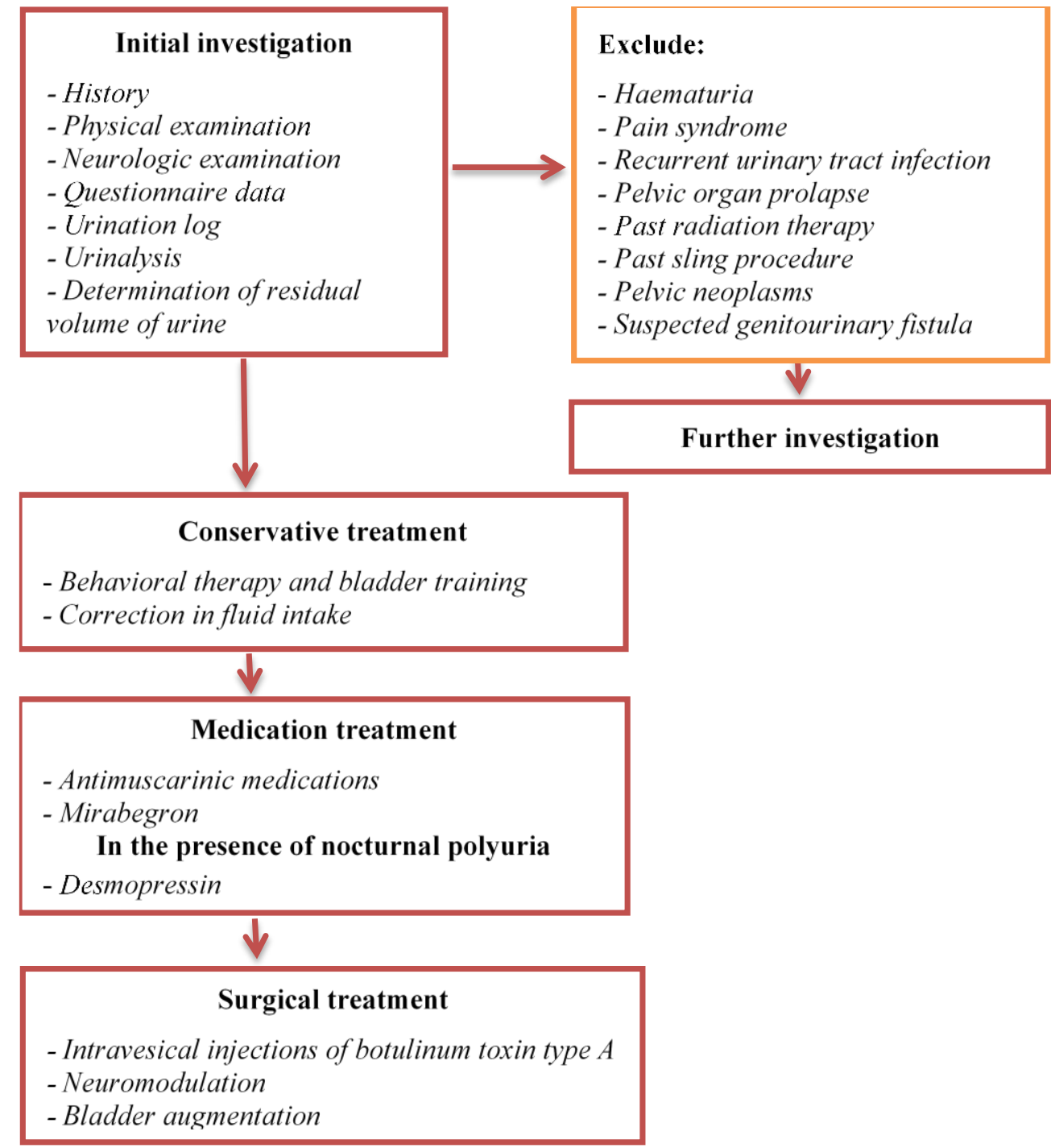

Fig. 1. An algorithm for the diagnosis and treatment of overactive bladder 


\section{REFERENCES}

1. Hubchev G., "Surgical tactitis in women with pelvic organ prolapse and latent stress incontinence"; Thesis 2017, pp. 25-31

2. Hubchev G., "Contemporary surgical treatment of stress incontinence in women"; Obstetrics and Gynecology, Book 5/2017, p. 62

3. Hubchev G., "Overactive bladder"; Scientific Work - Studios 2020, p.27

4. Ahlberg J., Edlund C., Wikkelso C. et al. Neurological signs are common in patients with urodynamically verified «idiopathic» bladder overactivity // Neurourol. Urodyn. - 2002. - Vol. 21. - N 1. - P. 65-70.

5. Gormley E.A., Lightner D.J., Faraday M., Vasavada S.P., American Urological Association, Society of Urodynamics et al. Diagnosis and treatment of overactive bladder (non-neurogenic) in adults: AUA/SUFU guideline amendment // J. Urol. - 2015. - May. - Vol. 193. - N 5. - P. 1572-1580.

6. Damiano R., Cicione A. The role of sodium hyaluronate and sodium chondroitin sulphate in the management of bladder disease // The Adv. Urol. - 2011. - Vol. 3. - N 5. - P. 223-232.

7. Stewart W.F., Van Rooyen J.B., Cundiff G.W., Abrams P., Herzog A.R., Corey R. et al. Prevalence and burden of overactive bladder in the United States // World J. Urol. - 2003. - May. - Vol. 20. - N 6. - P. 327-336.

8. Siracusano S., Cucchi S., Ciciliato S, Lampropoulou N, Vittur F. Urinary levels of giycosaminoglycans in patients with idiopathic detrusor overactivity // Int. Urogynecol. J. Pelvic. Floor. Dysfunct. - 2009. - Dec. Vol. 20. - N 12. - P. 1477-1480.

9. Vij M., Drake M.J. Clinical use of the $\beta 3$ adrenoceptor agonist mirabegron in patients with overactive bladder syndrome // Ther. Adv. Urol. - 2015. - Oct. - Vol. 7. - N 5. - P. 241-248.

10. Wein A.J., Rovner E.S. Definition and epidemiology of overactive bladder // Urology. - 2002. - Nov. - Vol. 60. - N 5. - Suppl. 1. - P. 7-12. - Discussion 12.

11. Zhang W., Song Y., He X. et al. Prevalence and risk factors of overactive bladder syndrome in Fuzhou Chinese women // Neurourol. Urodyn. - 2006. - Vol. 25. - N 7. - P. 717-721. 